\title{
DETERMINATION OF THE QUALITY OF a-Si:H FILMS: "TRUE" TRANSPORT PARAMETERS
}

\author{
N. Beck, A. Shah, N. Wyrsch, \\ Institute of Microtechnology, University of Neuchâtel, Rue Breguet 2, 2000 Neuchâtel, Switzerland, \\ tel. +41-38-20 54 52, fax.+41-38-25 42 76, e-mail: Beck@imt.unine.ch
}

\begin{abstract}
For the characterisation of a-Si:H layers, steady-state photoconductivity (SSPC) and steady-state photocarrier grating (SSPG) are currently used. But the $\mu \tau$-products deduced from these measurements are a function of the prevailing dangling bond occupation in the film and, thus, are not a measure of material quality. In the present paper the authors introduce the product $\mu^{0} \tau^{0}$, which is independent of dangling bond charge and which monitors material quality in terms of band mobility $\mu^{\circ}$ and capture time $\tau^{\circ}$, the latter being, in its turn, inversely proportional to dangling bond density. They present a simple evaluation procedure to deduce $\mu^{\circ} \tau^{\circ}$ from simultaneously performed SSPC and SSPG measurements. In undoped, but slightly n-type a-Si:H, the quality of the material will typically be related to the ambipolar diffusion length rather than to photoconductivity. The application of this method to a series of undoped a-Si:H films and solar cells incorporating these materials exhibited a good correlation between film properties and cell performances.
\end{abstract}

\section{INTRODUCTION}

Extensive research on the characterisation of a-Si:H films was carried out in the past with the aim to increase the basic understanding of a-Si: $\mathrm{H}$ material and, thus, improve the performance of a-Si:H based solar cells.

Nevertheless, a lack of quantitative correlation between the measured characteristics of a-Si:H films and cell performance persists [1], [2], [3]. In the present paper we contend that this lack of correlation is partly related to the type of methods normally used to characterise the transport properties of a-Si:H films. In fact, transport properties like photoconductivity and ambipolar diffusion length are measured, and these are strongly dependent on the occupation of dangling bond states. The dangling bond occupation in uniform a-Si:H films has little bearing to the (strongly non-uniform) distribution of dangling bond charge throughout the i-layer of a solar cell. Thus, simply transfering the results obtained from such transport measurements from films to solar cells must fail. Nevertheless, we will show in the present paper that the simultaneous measurement of both photoconductivity and ambipolar diffusion length, can indeed be a solution to this issue.

In fact, both electron and hole tranport, and, thus, both photoconductivity $\left(\sigma_{\mathrm{ph}}\right)$ and the ambipolar diffusion length $\left(L_{a m b}\right)$ are related to the same recombination centers (dangling bonds $\mathrm{D}^{\circ}, \mathrm{D}^{-}$and $\left.\mathrm{D}^{+}\right)[4]$. This correlated behaviour of $\sigma_{\mathrm{ph}}$ and $\mathrm{L}_{\mathrm{amb}}{ }^{2}$ enables one to determine the influence of the dangling bond charge on transport.

\section{"TRUE" TRANSPORT PARAMETERS: DEFINITIONS AND BASE FOR THEIR EVALUATION}

It is well known and widely accepted that the $\mu \tau$ products obtained by photoconductivity $\left(\sigma_{\mathrm{ph}}\right)$ and by the ambipolar diffusion length ( $L_{a m b}$ ) measurements are band mobility $x$ recombination time products $\left(\mu^{\mathrm{O}} \tau^{\mathrm{R}}\right)$ of the form:

$$
\begin{aligned}
\sigma_{p h} & =e \cdot G \cdot\left(\mu_{n}^{o} \tau_{n}^{R}+\mu_{p}^{o} \tau_{p}^{R}\right) \\
L_{a m b}^{2} & =\frac{k T}{e} \cdot \frac{\mu_{n}^{o} \tau_{n}^{R} \cdot \mu_{p}^{o} \tau_{p}^{R}}{\mu_{n}^{o} \tau_{n}^{R}+\mu_{p}^{o} \tau_{p}^{R}} \cdot C
\end{aligned}
$$

where $\mathrm{kT}$ is the thermal energy, e the elementary charge, $G$ the generation rate and $C$ a correction factor between 1 and 2 [5]. Combining both $\sigma_{\mathrm{ph}}$ and $L_{\text {amb }}$ measurements, allows one to determine the parameter $b=\mu_{n}^{0} \tau_{n}^{R} / \mu_{p}^{O} \tau_{p}^{R}=\mu_{n}^{O} n_{f} / \mu_{p}^{O} p_{f} ; b$ reflects the position of the two quasi Fermi-levels with respect to midgap [6]. b can be evaluated by an expression deduced from equations (1) and (2):

$$
\frac{b}{(b+1)^{2}}=\frac{L_{a m b}^{2} e^{2} G}{k T \sigma_{p h} C}
$$

The experimentally measured recombination times, as used in (1) and (2), are determined not only by the density of deep defects, but also by the dangling bond charge state. As dangling bond occupation is not a material characteristic but merely a function of experimental conditions, device structure and doping, it is not correct to directly use these $\mu^{\circ} \tau^{\mathrm{R}}$-products as a quality parameter for the material. However by measuring both, $\sigma_{\mathrm{ph}}$ and $\mathrm{L}_{\mathrm{amb}}$ we can define a $\mu \tau$-product which is a "true" quality parameter.

Thereby, we will take advantage of the fact that the transport properties of majority carriers (monitored by $\sigma_{\mathrm{ph}}$ ) and minority carriers (monitored by $L_{a m b}{ }^{2}$ ) are coupled via the dangling bonds, the latter being the common recombination centers for both of them [4]. Thus, electron and hole $\mu^{0} \tau^{R}$-products are not independent from each other, but display an anticorrelated behaviour (see Fig. 1). This behaviour makes it possible to evaluate and eliminate the contribution of the dangling bond occupation on $\mu^{0} \tau^{\mathrm{R}}$ measurements and, finally, to find an 


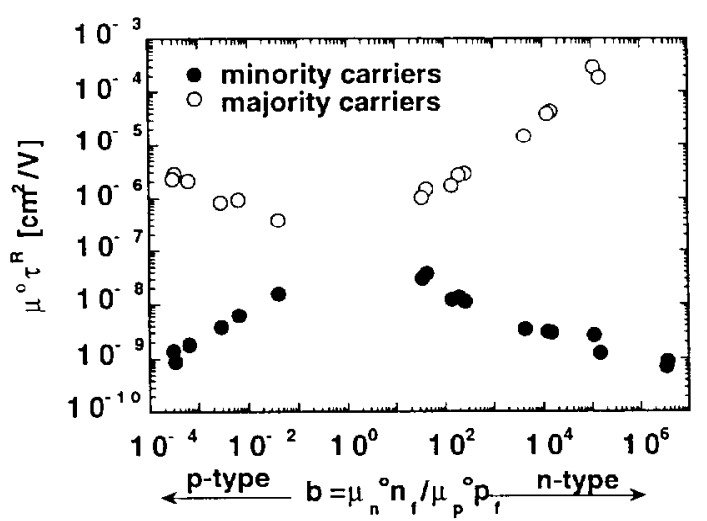

Fig.1. Anticorrelated change of majority and minority carrier $\mu^{o} \tau^{R}$-products with lowlevel doping, as measured by SSPC and SSPG.

$\mu^{0} \tau^{\mathrm{O}}$-product (band mobility $\times$ capture time) that is independent of dangling bond charge, and has the form:

$$
\mu_{n}^{0} \tau_{n}^{o}=\mu_{n}^{o} \frac{1}{v_{t h} N_{d b} \sigma_{n}^{0}} \quad \text { and } \quad \mu_{p}^{0} \tau_{p}^{\circ}=\mu_{p}^{0} \frac{1}{v_{t h} N_{d b} \sigma_{p}^{o}}
$$

Thus, the product $\mu^{0} \tau^{0}$ is a monitor for the deep defect density as well as for the band mobility, but it is independent of the dangling bond charge.

Consider now a dangling bond recombination model (DBR), which takes into account the three possible charge states of the dangling bonds: $D^{\circ}, D^{-}, D^{+}[7,8]$. Assume further a simplified description where recombination of free carriers only occurs through dangling bond states and where thermal emission from deep defects as well as recombination through bandtail states are neglected. The recombination times in $(1,2)$ are then:

$$
\begin{aligned}
& \tau_{n}^{R}=\frac{n_{f}}{r_{c n}^{O}+r_{c n}^{+}}=\left\{v_{t h} N_{d b} \sigma_{n}^{\circ}\left(f^{\circ}+\frac{\sigma_{n}^{+}}{\sigma_{n}^{\circ}} f^{+}\right)\right\}^{-1} \\
& \tau_{p}^{R}=\frac{p_{f}}{r_{c p}^{O}+r_{c p}^{-}}=\left\{v_{t h} N_{d b} \sigma_{p}^{\circ}\left(f^{\circ}+\frac{\sigma_{p}^{-}}{\sigma_{p}^{o}} f^{-}\right)\right\}^{-1},
\end{aligned}
$$

$r_{C n}{ }^{0}, r_{C n}+, r_{C P}{ }^{\circ}, r_{C P}$ are capture rates of free carriers on the different dangling bond states, $n_{f}, p_{f}$ densities of free electrons and holes, $\sigma_{n}{ }^{0}, \sigma_{n}{ }^{+}, \sigma_{p}{ }^{0}, \sigma_{p}{ }^{-}$capture cross-sections of dangling bonds, $N_{d b}$ the defect density, $v_{\text {th }}$ the thermal velocity and $f^{\circ}, f^{-}, f^{+}$the dangling bond occupation functions.

$f^{\circ}, f^{-}$and $f^{+}$in (5) and (6) are governed by the recombination traffic of free carriers $n_{f}$ and $p_{f}$ only and, thus, can be expressed as a function of them [8]:

$$
\begin{gathered}
f^{\circ}=1 /\left(\frac{p_{f}}{n_{f}} \frac{\sigma_{p}^{o}}{\sigma_{n}^{+}}+1+\frac{n_{f}}{p_{f}} \frac{\sigma_{n}^{o}}{\sigma_{p}^{-}}\right) \\
f^{-}=\frac{n_{f}}{p_{f}} \frac{\sigma_{n}^{o}}{\sigma_{p}^{-}} /\left(\frac{p_{f}}{n_{f}} \frac{\sigma_{p}^{o}}{\sigma_{n}^{+}}+1+\frac{n_{f}}{p_{f}} \frac{\sigma_{n}^{o}}{\sigma_{p}^{-}}\right)
\end{gathered}
$$

$$
f^{+}=\frac{p_{f}}{n_{f}} \frac{\sigma_{p}^{o}}{\sigma_{n}^{+}} /\left(\frac{p_{f}}{n_{f}} \frac{\sigma_{p}^{o}}{\sigma_{n}^{+}}+1+\frac{n_{f}}{p_{f}} \frac{\sigma_{n}^{o}}{\sigma_{p}^{-}}\right)
$$

Let us now introduce an additional hypothesis (10), which is not at all mandatory, but which can help the reader gain a better physical insight:

$$
\mu_{n}^{\circ} \tau_{n}^{\circ} \approx \mu_{p}^{\circ} \tau_{p}^{\circ}
$$

Hypothesis (10) can be justified on the basis of Time-ofFlight measurements performed by our group earlier $[9,10]$ on compensated and strongly degraded a-Si:H films, where almost all dangling bonds are neutral. The measurements (Fig. 2) show $\mu_{n}{ }^{0} \tau_{n}{ }^{0}$ and $\mu_{p}{ }^{0} \tau_{p}{ }^{0}$ to be almost equal $\left(\mu_{n}{ }^{0} \tau_{n}{ }^{\circ} / \mu_{p}{ }^{o} \tau_{p}=1.2\right)$. Note that the ratio given in the text of our previous papers $[9,10]$ was $\mu_{n}{ }^{\circ} \tau_{n}{ }^{o} / \mu_{p}{ }^{0} \tau_{p}{ }^{0}$ $=3 . .5$ : however, this value had not been correctly derived from the experimental data.

Thanks to hypothesis (10) we may now define a single quality parameter for a-Si:H material; we will call it $\mu^{\circ} \tau^{\circ}$ :

$$
\mu^{o} \tau^{o}=\mu_{n}^{0} \tau_{n}^{0}=\mu_{p}^{o} \tau_{p}^{0}
$$

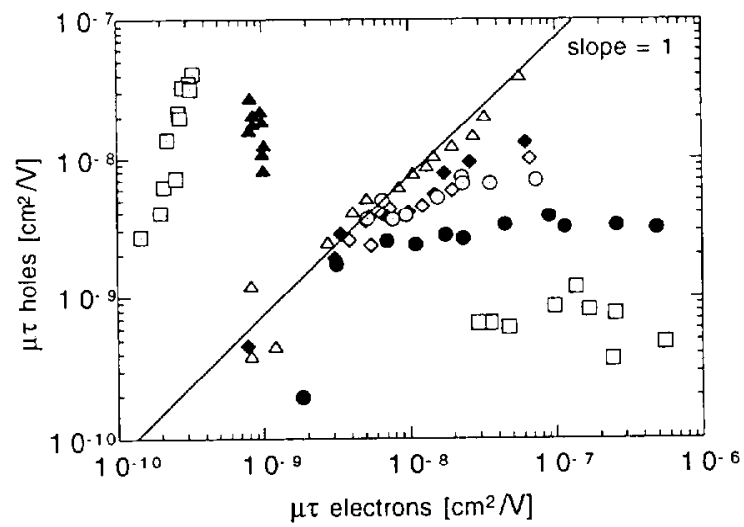

Fig. 2. Mutual change of electron and hole $\mu^{\circ} \tau$-products (see text) obtained by TOF measurements during degradation $[9,10]$. The measurements show the tendency of $\mu_{n}{ }^{o} \tau_{n}$ and $\mu_{p}{ }^{o} \tau_{p}$ to approach each other under light-soaking and then, when degrading further, to degrade with equal rates (line with slope 1).

\section{EVALUATION OF $\mu^{\circ} \tau^{O}$ FROM $\sigma_{p h}$ AND Lamb}

Starting with the expressions for the experimentally acessible recombination times (5), (6), and using the dangling bond occupation functions $(7),(8),(9)$ and introducing the second hypothesis (10) in the calculation, $\mu^{0} \tau^{\circ}$ can now be deduced. The following results are obtained:

$$
\mu_{n}^{o} \tau_{n}^{R}=\mu^{o} \tau^{o} \frac{b}{b+1}\left(\frac{\sigma_{n}^{o}}{\sigma_{n}^{+}} \frac{1}{b}+1+\frac{\sigma_{p}^{o}}{\sigma_{p}^{-} b}\right)
$$




$$
\mu_{p}^{o} \tau_{p}^{R}=\mu^{\circ} \tau^{\circ} \frac{1}{b+1}\left(\frac{\sigma_{n}^{o}}{\sigma_{n}^{+}} \frac{1}{b}+1+\frac{\sigma_{p}^{o}}{\sigma_{p}^{-}} b\right)
$$

The expressions (12) and (13) show that the measured $\mu^{\mathrm{D}} \tau^{\mathrm{R}}$-products generally need to be corrected by a certain factor, which is a function of the parameter $b$ and, thus, is influenced by doping (also by low-level, non-intentional doping due e.g. to [O] contamination). Nevertheless, there is a problem with these expressions: they cannot be applied to "truly" intrinsic (i.e. "compensated" or "midgap") a-Si:H, where both electrons and holes contribute to the photoconductivity. Thus, it is more convenient rewrite the expression for photoconductivity (1) and obtain:

$$
\frac{\sigma_{p h}}{e G}=\mu^{0} \tau^{0} \cdot z \text { with } z=\left(\frac{\sigma_{n}^{o}}{\sigma_{n}^{+}} \frac{1}{b}+1+\frac{\sigma_{p}^{o}}{\sigma_{p}^{-}} b\right)
$$

This formula is now valid for all types of a-Si: $\mathrm{H}$; the character of the material is monitored by the correction factor z. Furthermore, it is not necessary to know the different contributions of electrons and holes to $\sigma_{\mathrm{ph}}$. The information gained by $L_{a m b}$ measurements appears here only through the parameter $b$.

Let us now evaluate $\mu^{0} \tau^{0}$ for some particular situations:

When the material is "truly intrinsic" and the Fermi energy level is near midgap (i.e. in compensated a-Si:H), $b$ becomes close to unity and the correction factor $z$ approaches 1. Thus, $\mu^{0} \tau^{R}$ (deduced from $\sigma_{p h}$ ) and $\mu^{0} \tau^{0}$ become identical. In this case, $\sigma_{\mathrm{ph}}$ is the proper parameter to describe material quality.

On the other hand side, in n-type material (even in undoped slightly n-type films) $b " 1$ and the correction factor takes on the value $z \approx b\left(\sigma_{p}{ }^{\circ} / \sigma_{p}{ }^{-}\right)$. In this situation, according to equation (13), $\mu^{0} \tau^{\circ}$ becomes proportional to $\mu_{p}{ }^{0} \tau_{p}{ }^{R}$. This means, that for $n$-type a-Si: $\mathrm{H}$ with $b>\sigma_{p}{ }^{-1} \sigma_{p}{ }^{\circ}$, material quality is monitored by the ambipolar diffusion

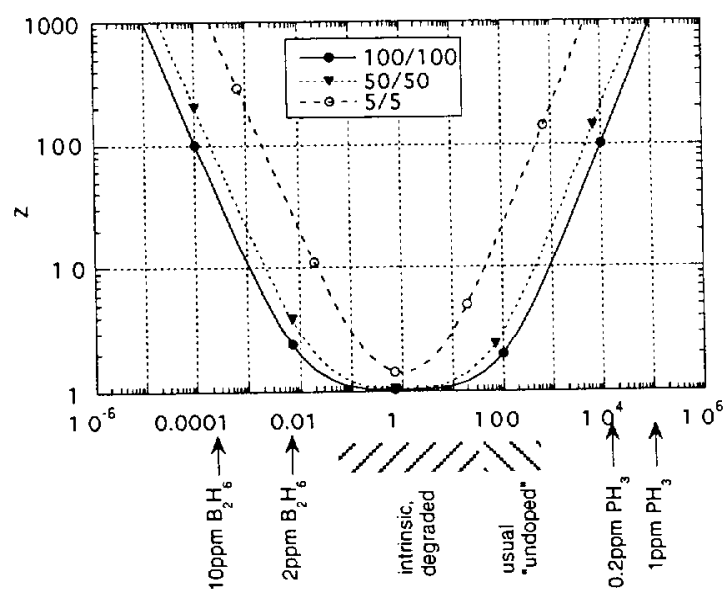

Fig. 3. Calculated magnitude of the correction factor $z$ for experimental $\mu^{o} \tau^{A}$-products deduced from $\sigma_{p h}$ for different values of $b$. The insert indicates the three different ratios of capture cross sections $\sigma_{n}{ }^{+} / \sigma_{n}{ }^{0}$ and $\sigma_{p}{ }^{-} / \sigma_{p}{ }^{a}$ used. On the $x$ axis some typical values of $b$ are indicated. length and not by $\sigma_{p h}$. The same also applies to p-type aSi:H. Thereby the capture cross section ratios $\sigma_{n}{ }^{+} / \sigma_{n}{ }^{\circ}$ and $\sigma_{p}{ }^{-} / \sigma_{p}{ }^{\circ}$, when compared to the experimental parameter $b$, will mark the limits between intrinsic a-Si:H and n-type or $p$-type material. Unfortunately, the values of $\sigma_{n}{ }^{+} / \sigma_{n}{ }^{\circ}$ and $\sigma_{p} / \sigma_{p}{ }^{\circ}$ are still a controversial topic; values given in literature vary between 1.5 [11] and $\geq 100$ [12]. Here, we will use for the evaluation of $\mu^{0} \tau^{0}$-products, $\sigma_{n}{ }^{1} / \sigma_{n}{ }^{0}=\sigma_{p}$ $/ \sigma_{p}{ }^{\circ}=50$. This estimate is based on the behaviour of $\mu^{0} \tau^{0}$ products with degradation and doping (to be presented elsewhere).

Fig.3. shows the calculated magnitude of the correction factor $z$ for the parameter $\mu^{0} \tau^{R}$ deduced from $\sigma_{p h}$, plotted as a function of $b$. "Typical" values for $b$, as obtained by low-level $\mathrm{PH}_{3}$ and $\mathrm{B}_{2} \mathrm{H}_{6}$ doping are also indicated.

\section{CORRELATION BETWEEN $\mu^{\circ} \tau^{\circ}-$ PRODUCTS OF a-Si:H FILMS AND SOLAR CELL PERFORMANCES}

The quality-related parameter $\mu^{0} \tau^{0}$ was mainly introduced by us with the aim to correlate film properties and cell performances. For that purpose, a series of films and cells was produced by the VHF-GD deposition technique at 70 $\mathrm{MHz}$ [13], while varying thereby the deposition temperature for the undoped layer. For each film - cell couple, first a $\sim 2.5 \mu \mathrm{m}$ thick, undoped a-Si: $\mathrm{H}$ film was deposited on a glass substrate; subsequently, using the same set of deposition parameters, the "same" a-Si:H material was incorporated as an i-layer in a standard, $\sim 0.6$ $\mu \mathrm{m}$ thick $\mathrm{p}-\mathrm{i}-\mathrm{n}$ solar cell. Before measuring the characteristics, both cells and films were annealed for 2 hours at $180^{\circ} \mathrm{C}$. The a-Si:H films were then characterised by SSPG, SSPC, Photothermal Deflection Spectroscopy (PDS), Constant Photocurrent Method (CPM) [14] and dark conductivity measurements. For the transport measurements, a Krypton laser $\left(\lambda=647 \mathrm{~nm}\right.$ at $3 \mathrm{~mW} / \mathrm{cm}^{2}$ to $90 \mathrm{~mW} / \mathrm{cm}^{2}$ ) was used, in order to obtain, in the film, a generation rate of electron hole pairs of $2-60 \cdot 10^{19} 1 / \mathrm{cm}^{3} \mathrm{~s}$. For the cells, the efficiency $(\eta)$ were detemined, under standard AM1.5 illumination at $100 \mathrm{~mW} / \mathrm{cm}^{2}$, using a twosource solar simulator (Wacom WXS-140S-10).

\section{The Annealed State}

The $\mu^{\circ} \tau^{\circ}$-products evaluated for this series of films as well as the corresponding solar cell efficiencies are plotted in Fig. 4 . The quality of the film material, monitored by $\mu^{0} \tau^{\circ}$, is almost constant for temperatures above $220^{\circ} \mathrm{C}$. Lowering the deposition temperature leads to a deterioration of material quality. Most films exhibited a clear extrinsic behaviour in the annealed state.

Cell efficiencies slightly peak at $220^{\circ} \mathrm{C}$ and exhibit the same strong decrease of quality with lower deposition temperatures. As the reduction of optical absorption in the device due to the increased bandgap $\left(E_{\mathrm{g}} \approx 1.76 \mathrm{eV}\right.$ from Tauc's plot for $T_{\text {set }}=150^{\circ} \mathrm{C}$ compared to $E_{\mathrm{g}}=1.73 \mathrm{eV}$ for $\mathrm{T}_{\text {set }}=270^{\circ} \mathrm{C}$ ) can be estimated to be less than $5 \%$., the observed deterioration of the cells for low deposition temperatures can mainly be attributed to the bad quality of the material. On the other hand, the slight reduction of cell efficiencies for higher deposition temperatures is not consistent with the observed good film quality. As for these cells the value of $V_{o c}$ is also lower, chemical and 


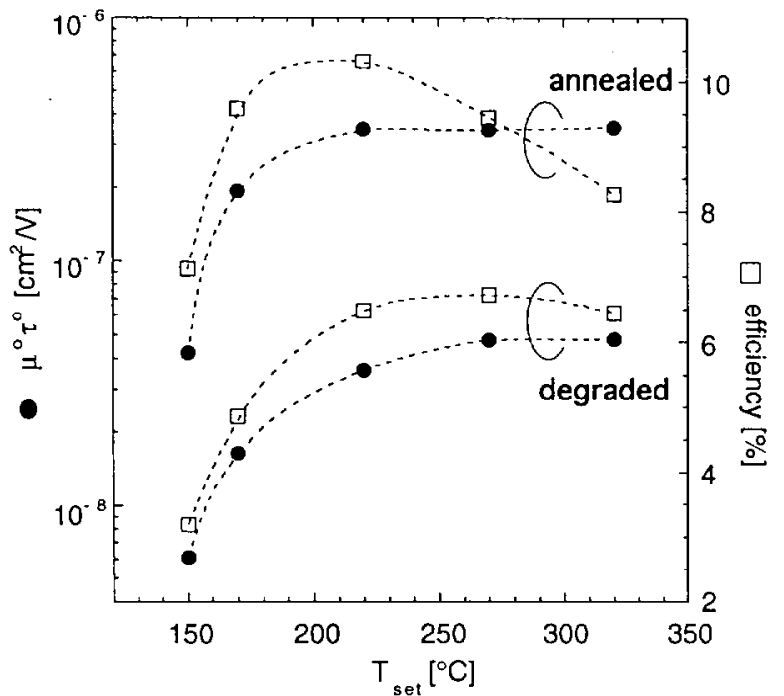

Fig. $4 . \mu^{\circ} \tau^{\circ}$-products evaluated on a series of $2.5 \mu \mathrm{m}$ thick, undoped films and efficiencies of solar cells incorporating the "same" films as an i-layer, in the annealed state and the degraded state. $T_{\text {set }}$ is the setpoint of the deposition temperature. (For evaluation we assumed $\sigma_{n}{ }^{+} / \sigma_{n}{ }^{0}=\sigma_{p}{ }^{-} / \sigma_{p}{ }^{0}=50$ ).

structural problems (e.g. interdiffusion or interface deterioration) may be responsible for coll deterioration.

\section{The degraded state}

The cells were degraded during 3 weeks by an AM1.5 light source of $100 \mathrm{~mW} / \mathrm{cm}^{2}$ (array of Philips $P L-L$ $24 \mathrm{~W} / 95 / 4 \mathrm{P}$ lamps) at $47^{\circ} \mathrm{C}$. The films were light-soaked during 4 weeks by a 6 -sun high pressure sodium lamp (spectral maximum at $590 \mathrm{~nm}$ with a FWHM of around $40 \mathrm{~nm}$ ) at $46^{\circ} \mathrm{C}$. Though films and cells were light-soaked by different light sources, they show a very good correlation after light-soaking (see fig. 4): both exhibit the same strong deterioration of quality for lower deposition temperatures. Thereby, the quality of a-Si:H films was reduced by a factor of $\approx 10$ and cell efficiencies by $20 \%$ $50 \%$. After degradation, all films showed to be almost intrinsic.

\section{CONCLUSIONS}

We have introduced and defined a new $\mu^{\circ} \tau^{\circ}$-product which can be used as a quality parameter for a-Si:H material optimisation. $\mu^{0} \tau^{0}$ is determined by band mobility and deep defect density. This quality-related $\mu^{0} \tau^{0}$ product does not depend - in contrast with direct experimental data - on the dangling bond occupation prevailing in the material. Furthermore, we have shown that $\mu^{\circ} \tau^{0}$ can easily be determined from SSPC and SSPG measurements according to the following procedure: first, and most important in order to get a correct information about the material quality, one has to identify if the material is intrinsic (midgap) or if it has an extrinsic character ( $n$-type or p-type). Thereby, the following criterion for the experimentally determined parameter $b$ applies:

$$
\begin{array}{ll}
\text { 1) intrinsic (midgap): } & \frac{\sigma_{n}^{\circ}}{\sigma_{n}^{+}}<b<\frac{\sigma_{p}^{-}}{\sigma_{p}^{\circ}} \\
\text { 2) extrinsic: } & b>\frac{\sigma_{p}^{-}}{\sigma_{p}^{\circ}} \text { or } b<\frac{\sigma_{n}^{\circ}}{\sigma_{n}^{+}}
\end{array}
$$

If the material fits into category 1), the quality is simply given by the $\mu \tau$ product deduced from photoconductivity: $\mu^{\circ} \tau^{\circ}=\sigma_{\mathrm{ph}} /(\mathrm{eG})$. On the other hand, if condition 2) is fullfilled, the material quality is monitored by the ambipolar diffusion length $L_{a m b}$. In this case, $L_{a m b}$ can be related to $\mu^{0} \tau^{0}$ by the following expression $\left(\sigma_{p} \pm / \sigma_{p}{ }^{0} \approx 50\right)$ :

$$
\mu^{\circ} \tau^{\circ}=L_{\text {amb }}^{2} \frac{e}{k T} \frac{1}{C} \frac{\sigma^{ \pm}}{\sigma^{\circ}} \text { with } C \approx 1.5 \text { and } \sigma_{p}^{ \pm} / \sigma_{p}{ }^{o} \approx 50
$$

In limit cases, where the sample can neither clearly be attributed to 1) nor 2), the general expression (14) must be used in order to obtain $\mu^{\circ} \tau^{\circ}$.

When applying $\mu^{0} \tau^{\circ}$ to correlate film quality and cell performances one finds: the proposed quality parameter $\mu^{\circ} \tau^{\circ}$ correlates in general well with the measured cell performances, above all in the degraded state. However, this correlation may be "hidden" in certain cases by more severe problems in the solar cell structure, which are not related to the quality of the intrinsic layer.

\section{ACKNOWLEDGMENTS}

The authors acknowledge the help of $S$. Dubail for sample preparation, as well as financial support from the Swiss National Science Foundation under grant (FN-39377) and from the Swiss Federal Renewable Energy Program under mandat (EF-REN(93)032).

\section{REFERENCES}

[1] C. Wronski et al., MRS Proc. 297, 1993, p. 443.

[2] R. Schropp et al., J. Non-Cryst. Sol. 164-166, 1993, p. 709.

[3] J. Yang et al., MRS Proc. 1994, to be published.

[4] A. Shah et al., J. Non-Cryst. Sol. 164-166, 1993, p. 485 .

[5] E. Sauvain, Ph. D. Thesis, University of Neuchâtel, 1992.

[6] P. Pipoz et al., MRS Proc. 258, 1992, p. 777

[7] F. Vaillant et al., Phys. Rev. B 34, 1986, p. 4088.

[8] J. Hubin et al., Phil. Mag. Lett. 66, 1992, p. 115.

[9] N. Beck et al., Proc. 11th ECPVSEC, 1992, p. 625.

[10] N. Beck et al., MRS Proc. 297, 1993, p. 479.

[11] K. Hattori et al., J. Non-Cryst. Sol.137\&138, 1991, p. 363.

[12] N. Wyrsch et al., J. Non-Cryst. Sol.137\&138, 1991, p. 431.

[13] H. Curtins et al., Plasma Chem. \& Plasma Proc. 7, 1987 , p. 267.

[14] M. Vanecek et al., Solid State Com. 39, 1981, p. 1199 . 